\title{
Entorhinal and Transentorhinal Atrophy in Preclinical Alzheimer's Disease
}

\author{
Sue Kulason ${ }^{1,2,3 *}$, Eileen X ${ }^{1}$, Daniel J. Tward ${ }^{4,5}$, Arnold Bakker ${ }^{6,7}$, Marilyn Albert ${ }^{7}$, \\ Laurent Younes ${ }^{1,2,8}$ and Michael I. Miller ${ }^{1,2,3,9}$ for the Alzheimer's Disease \\ Neuroimaging Initiative \\ ${ }^{1}$ Center for Imaging Science, Johns Hopkins University, Baltimore, MD, United States, ${ }^{2}$ Institute for Computational Medicine, \\ Johns Hopkins University, Baltimore, MD, United States, ${ }^{3}$ Department of Biomedical Engineering, Johns Hopkins University, \\ Baltimore, MD, United States, ${ }^{4}$ Department of Computational Medicine, University of California, Los Angeles, Los Angeles, \\ CA, United States, ${ }^{5}$ Department of Neurology, Ahmanson-Lovelace Brain Mapping Center, University of California, Los \\ Angeles, Los Angeles, CA, United States, ${ }^{6}$ Department of Psychiatry and Behavioral Sciences, Johns Hopkins University, \\ Baltimore, MD, United States, ${ }^{7}$ Department of Neurology, Johns Hopkins University, Baltimore, MD, United States, \\ ${ }^{8}$ Department of Applied Mathematics and Statistics, Johns Hopkins University, Baltimore, MD, United States, ${ }^{9}$ Kavli \\ Neuroscience Discovery Institute, Johns Hopkins University, Baltimore, MD, United States
}

\section{OPEN ACCESS}

Edited by:

Jie Tu,

Chinese Academy of Sciences, China

Reviewed by:

Ricardo Insausti,

University of Castilla-La Mancha,

Spain

José Luna-Muñoz,

Universidad Nacional Autonóma de

México, Mexico

*Correspondence:

Sue Kulason

skulaso1@jhu.edu

Specialty section: This article was submitted to

Neurodegeneration,

a section of the journal

Frontiers in Neuroscience

Received: 14 April 2020

Accepted: 09 July 2020

Published: 21 August 2020

Citation:

Kulason S, Xu E, Tward DJ, Bakker A, Albert $M$, Younes $L$ and Miller $M I$ (2020) Entorhinal and Transentorhinal Atrophy in Preclinical Alzheimer's Disease. Front. Neurosci. 14:804. doi: 10.3389/fnins.2020.00804
This study examines the atrophy patterns in the entorhinal and transentorhinal cortices of subjects that converted from normal cognition to mild cognitive impairment. The regions were manually segmented from $3 \mathrm{~T} \mathrm{MRI}$, then corrected for variability in boundary definition over time using an automated approach called longitudinal diffeomorphometry. Cortical thickness was calculated by deforming the gray matter-white matter boundary surface to the pial surface using an approach called normal geodesic flow. The surface was parcellated based on four atlases using large deformation diffeomorphic metric mapping. Average cortical thickness was calculated for (1) manually-defined entorhinal cortex, and (2) manually-defined transentorhinal cortex. Group-wise difference analysis was applied to determine where atrophy occurred, and change point analysis was applied to determine when atrophy started to occur. The results showed that by the time a diagnosis of mild cognitive impairment is made, the transentorhinal cortex and entorhinal cortex was up to $0.6 \mathrm{~mm}$ thinner than a control with normal cognition. A change point in atrophy rate was detected in the transentorhinal cortex 9-14 years prior to a diagnosis of mild cognitive impairment, and in the entorhinal cortex 8-11 years prior. The findings are consistent with autopsy findings that demonstrate neuronal changes in the transentorhinal cortex before the entorhinal cortex.

Keywords: transentorhinal, entorhinal, preclinical, cortical thickness, change point, diffeomorphometry

\section{BACKGROUND}

Evidence suggests that neuropathological changes of Alzheimer's disease (AD) begin years before the onset of clinical symptoms (Sperling et al., 2011). Accumulation of these neuropathological changes is associated with neuronal injury, which can be measured indirectly by structural magnetic resonance imaging (MRI) (Atiya et al., 2003; Kantarci and Jack, 2004). A number of MRI studies have detected atrophy in the entorhinal cortex (ERC), hippocampus and amygdala associated with clinical disease severity (Devanand et al., 2007; La Joie et al., 2012; Miller et al., 2015b) and years 
to $\mathrm{AD}$ dementia conversion (Atiya et al., 2003; Kantarci and Jack, 2003). More recent MRI studies have focused on evidence of atrophy that precede clinical symptoms (Jack et al., 2004; Csernansky et al., 2005; den Heijer et al., 2006; Apostolova et al., 2010; Dickerson et al., 2011; Miller et al., 2013; Soldan et al., 2015; Pettigrew et al., 2016), often detecting these smaller changes using time-series data analysis (Durrleman et al., 2012) and survival analysis. These MRI biomarkers of $\mathrm{AD}$-related atrophy prior to manifestation of clinical symptoms are of interest because (1) they may aid in assessing efficacy of therapeutic interventions and (2) they may aid in the identification of populations that can benefit from therapeutic intervention prior to clinical symptoms.

Braak's staging of $\mathrm{AD}$ suggests that cortical accumulation of neurofibrillary tangles starts in the transentorhinal cortex (TEC), spreads medially to the ERC, and then involves the hippocampus and amygdala (Braak and Braak, 1991; Braak et al., 2006). Accumulation of tau pathology has been correlated with changes in cognitive status in the presence of $\beta$-amyloid plaques (Nelson et al., 2012), as well as with TEC atrophy as detected in MRI (Xie et al., 2018). While relatively few MRI studies exist on AD-related TEC changes (Tward et al., 2017b; Wolk et al., 2017), our recent work demonstrated that subjects with mild cognitive impairment (MCI) have increased baseline atrophy and increased rate of atrophy compared to cognitively normal controls, and that changes in the TEC had greater magnitude than changes in the ERC, hippocampus, and amygdala (Kulason et al., 2019). In addition, our group's work on change point analysis has demonstrated MRI-based shape metrics can detect atrophy in the ERC earlier than hippocampal and amygdalar atrophy (Younes et al., 2014). These changes precede clinical symptoms by up to 10 years.

The evidence of MRI biomarkers that precede clinical symptoms taken together with evidence of cortical AD-related changes selectively occurring first in the TEC motivate a close look at TEC atrophy over the preclinical stage of $\mathrm{AD}$ progression. In this study, we aim to localize, both spatially and temporally, MRI-based atrophy detection within the TEC and ERC by examining subjects from two diagnostic groups: stable normal cognition (NC), and NC to MCI converters.

\section{METHODS}

\subsection{Data Collection}

Subjects were selected from the ADNI database (adni.loni.usc.edu). The criteria for stable NC included the absence of a diagnosis of MCI or $\mathrm{AD}$ on all baseline and follow-up visits, a CDR score of 0 on all baseline and follow-up visits, evidence of performance within the normal range on the Logical Memory Subtest of the Wechsler Memory Scale on all baseline and follow-up visits (based on education adjusted norms), and negative results for elevated amyloid $\beta$ levels on the baseline visit (greater than a cut off of $192 \mathrm{pg} / \mathrm{mL}$ from CSF as established by the ADNI Biospecimen Core).

The criteria for NC to MCI converters included evidence of performance within the normal range on the Logical Memory Subtest of the Wechsler Memory Scale at baseline (based on education adjusted norms), a CDR score of 0 on the baseline
TABLE 1 | Demographics (mean \pm standard deviation where applicable).

\begin{tabular}{lcc}
\hline Diagnostic group & Stable NC & NC to $\mathbf{~ M C l}$ \\
\hline Sample size (n) & 33 & 17 \\
Baseline age (years) & $72.3 \pm 5.5$ & $74.9 \pm 5.3$ \\
Sex (\% Female) & 45.5 & 70.6 \\
$\#$ of scans (years) & $4.5 \pm 0.6$ & $4.6 \pm 1.1$ \\
Scan period (years) & $3.4 \pm 1.1$ & $2.9 \pm 1.0$ \\
Clinical evaluation period (years) & $5.3 \pm 2.4$ & $6.4 \pm 3.7$ \\
\hline
\end{tabular}

exam, a diagnosis of NC at baseline, and a diagnosis of MCI or dementia at a subsequent follow-up visit. Estimated MCI age-ofonset was established based on annual assessment of diagnosis. Note that subjects missing a diagnostic evaluation more than a year prior to MCI diagnosis were excluded, and one subject with an MCI diagnosis was also excluded due to a stable, high score on the Logical Memory Subtest of the Weschler Memory Scale 5 years after diagnosis.

In addition, subjects had to have a minimum of three $3 \mathrm{~T}$ MRI scans over 2 or more years. Out of the 30 subjects that met all criteria for NC to MCI converters, all subjects were examined and 17 had a continuous collateral sulcus (CoS) and were included in this study (see section 2.2 for detailed explanation). We examined a subset of available stable NC subjects to reach a total sample size of 50 . Out of the 84 subjects that met all criteria for stable NC, 68 were examined and 33 had a continuous collateral sulcus and were included in this study. The demographics of subjects included in this study are summarized in Table 1. Two-sample t-tests showed no significant diagnostic group differences by age, number of scans, scan period, or clinical evaluation period. Pearson chi-squared test showed no significant diagnostic group difference by sex. Note that the clinical evaluation period is longer than the scan period because the scan protocol was updated in the ADNI 3 cohort to an accelerated scan sequence. These accelerated scans were not included in this analysis.

\subsection{Manual Segmentation and Surface-Based Morphometry}

As in previous projects (Tward et al., 2017b; Kulason et al., 2019), we restricted the analysis to the left hemisphere and excluded subjects with a discontinuous $\operatorname{CoS}$ in the defined region of interest. There are several variants of the $\operatorname{CoS}$ to consider, illustrated in Figure 1. The first variant is a deep, continuous sulcus where the rhinal sulcus shares a sulcal bed with the collateral sulcus proper. This variant has been referred to as Type I CoS (Ding and Van Hoesen, 2010) and Type II/Type III rhinal sulcus (Huntgeburth and Petrides, 2012). The second variant is a discontinuous $\mathrm{CoS}$ where the collateral sulcus proper begins posterior to the GI. This variant has been referred to as a Type IIa CoS (Ding and Van Hoesen, 2010) and Type I rhinal sulcus (Huntgeburth and Petrides, 2012). Finally, there is a variant with a discontinuous $\mathrm{CoS}$ where the collateral sulcus proper begins anterior to the GI, and therefore is excluded for this study. This variant has been referred to as a Type IIb CoS 

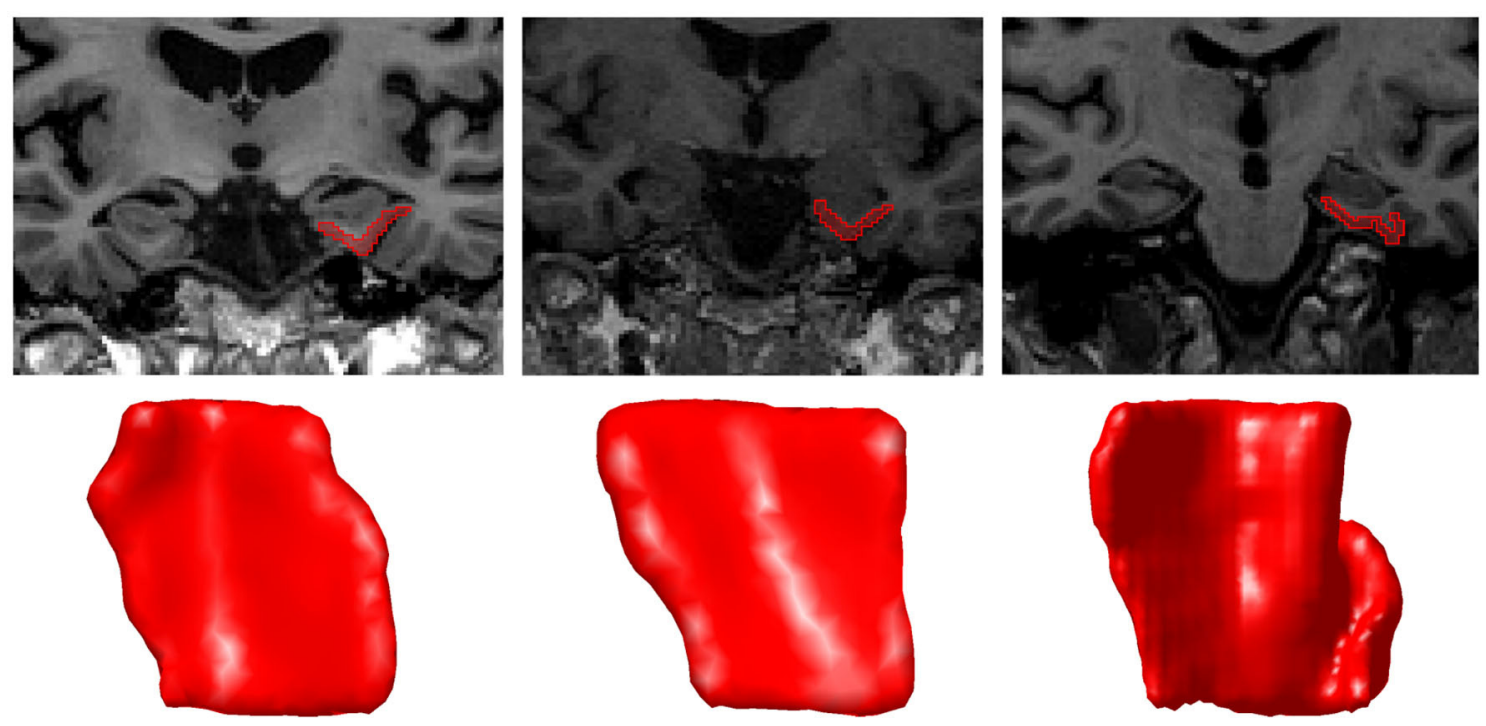

FIGURE 1 | A coronal MRI section and corresponding surface of each CoS variant Type I (left), Type Ila (middle), and Type Ilb (right). In Type Ilb, the segmentation was extended to the second CoS, also known as the collateral sulcus proper. The orientation of surfaces is as follows: anterior (top), posterior (bottom), medial (left), lateral (right).

TABLE 2 | Distribution of collateral sulcus variants.

\begin{tabular}{lccc}
\hline & $\begin{array}{c}\text { Type I } \\
\text { (continuous CoS) }\end{array}$ & $\begin{array}{c}\text { Type Ila } \\
\text { (discontinuous } \\
\text { posterior CoS) }\end{array}$ & $\begin{array}{c}\text { Type Ilb } \\
\text { (discontinuous } \\
\text { anterior CoS) }\end{array}$ \\
\hline $\mathrm{NC}(n=68)$ & $21(31 \%)$ & $12(18 \%)$ & $35(51 \%)$ \\
$\mathrm{NC}$ to $\mathrm{MCl}(n=30)$ & $13(43 \%)$ & $4(13 \%)$ & $13(43 \%)$ \\
Total $(n=98)$ & $34(35 \%)$ & $16(16 \%)$ & $48(49 \%)$ \\
\hline
\end{tabular}

Type Ilb CoS were excluded from this analysis.

(Ding and Van Hoesen, 2010) and also falls into the category for Type I rhinal sulcus (Huntgeburth and Petrides, 2012). Table 2 categorizes the proportion of $\mathrm{CoS}$ variants prior to exclusion of Type IIb. Previous work has shown there is a relationship between CoS depth and the boundaries of the ERC and TEC with respect to anatomical markers (Insausti et al., 1998). Briefly, in a shallow $\operatorname{CoS}(<1 \mathrm{~cm})$ the ERC extends to the deepest extent of the CoS. In a regular CoS (between 1 and $1.5 \mathrm{~cm}$ ), the ERC extends to the midpoint of the medial bank of the CoS. In a deep $\operatorname{CoS}(>1.5 \mathrm{~cm})$ the ERC extends up to the CoS. In this subject set we found that excluded Type IIb CoS subjects most often had a shallow CoS, while the Type I and Type IIa variants included in this study were of a regular to deep CoS type.

$2263 \mathrm{~T}$ T1 MRI scans were used in this study. ERC and TEC were segmented manually using Seg3D software (Center for Integrative Biomedical Computing, 2016). We followed an established procedure for segmentation and delineation of the ERC and TEC (Tward et al., 2017b) that was based on anatomical landmarks described near cytoarchitectonically-defined ERC boundaries (Insausti et al., 1998; Ding and Van Hoesen, 2010).
The anterior boundary of the ERC and TEC were defined $4 \mathrm{~mm}$ anterior to the hippocampal head. Delineation of the ERC and TEC anterior to this boundary is more complex and excluded from this study. Earlier works suggest that the area anterior to this region is a mix of ERC and perirhinal cortex (Insausti et al., 1998), or olfactory cortex (Krimer et al., 1997), whereas a more recent work suggests that this area is, in fact, part of the ERC (Ding and Van Hoesen, 2010). The posterior boundary for ERC and TEC was defined $2 \mathrm{~mm}$ posterior to the gyrus intralimbicus (GI) (Insausti et al., 1998). The medial extent of the ERC was defined as far as the gray/white boundary was visible. This delineation excludes a small dorsal medial aspect of the ERC that rests against the amygdala, and is similar to how other T1 MRI protocols delineate the ERC (Desikan et al., 2006; Maass et al., 2015). The lateral extent of the ERC and medial extent of the TEC was defined at the medial extent of the collateral sulcus (CoS), as is found in a deep CoS (Insausti et al., 1998). The lateral extent of the TEC was defined as being at the deepest extent of the CoS, as is also found in a deep CoS (Ding and Van Hoesen, 2010).

Figure 2 shows a sample of ERC plus TEC surfaces generated from manual segmentations. A population template for the ERC plus TEC surface was calculated from these surfaces by taking the average (Fréchet mean) diffeomorphism in a Bayesian setting (Ma et al., 2008). Then, the segmentations were adjusted for variability in boundaries over time by mapping the population template simultaneously onto each scan of a time series (Tward et al., 2017a). The result is a set of surfaces of the ERC plus TEC.

\subsection{Surface Parcellation}

Studies of this region have tended to use inconsistent nomenclature. We mapped four atlases with commonly used 

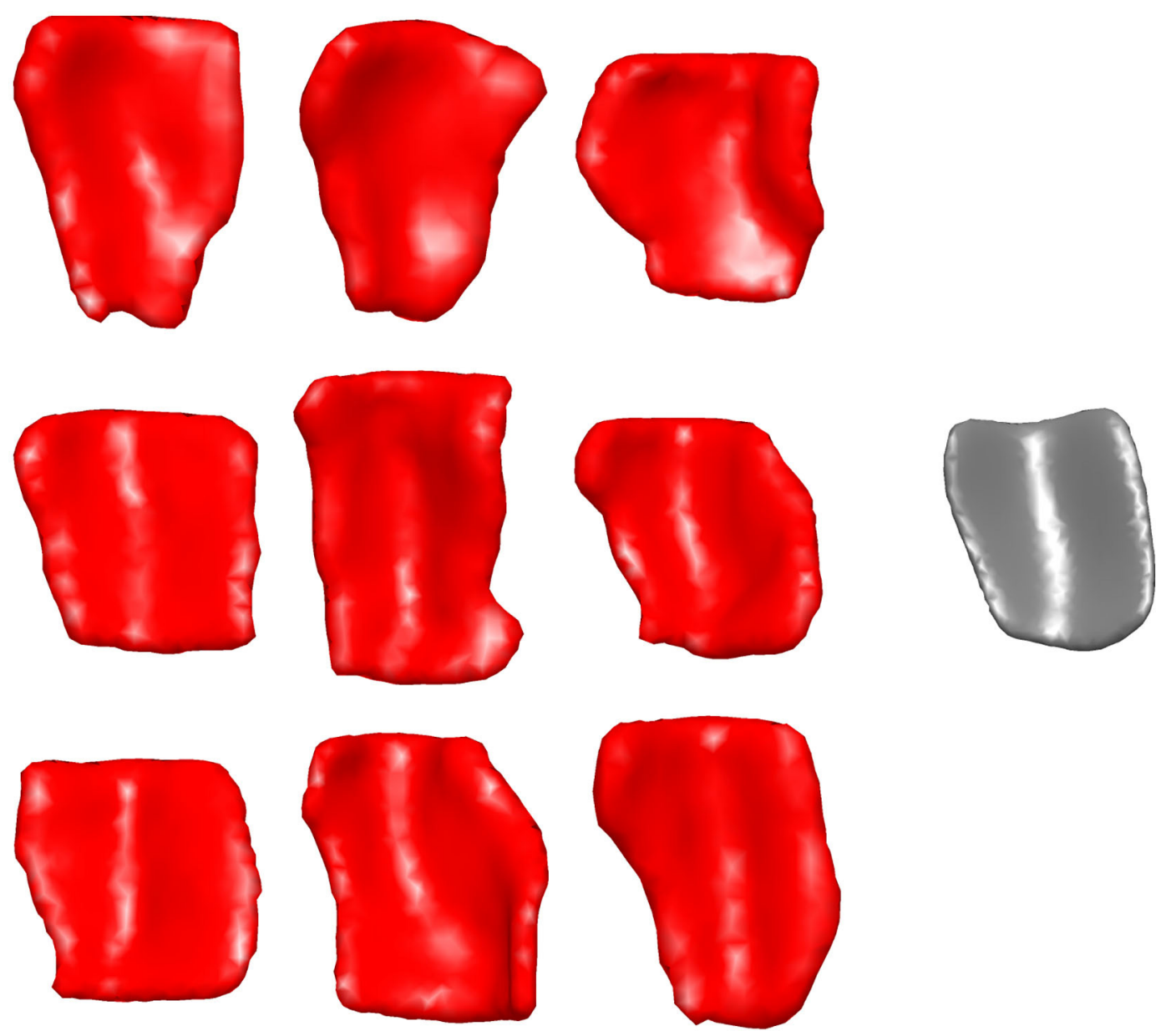

FIGURE 2 | Sample of ERC plus TEC surfaces generated from manual segmentations (left) and the resultant population template (right). The orientation is as follows: anterior (top), posterior (bottom), medial (left), lateral (right).

sub-regional labels onto our ERC plus TEC population template: (1) manual labels of ERC and TEC based on cortical folding seen in structural MRI (Tward et al., 2017b), (2) automated labels of ERC and parahippocampal gyrus (PHG) based on cortical folding seen in structural MRI (Desikan et al., 2006), (3) labels of posterior medial ERC (pmERC), anterior lateral ERC (alERC), and perirhinal cortex (PRC) based on connectivity patterns seen in functional MRI after manual segmentation of ERC in structural 7T MRI (Maass et al., 2015), and (4) histological labels of intermediate superior ERC, intermediate rostral ERC, intermediate caudal ERC, prorhinal ERC, medial rostral ERC, medial caudal ERC, lateral ERC, sulcal ERC and TEC as identified in an 11T ex vivo MRI (Krimer et al., 1997; Miller et al., 2015a).

Manual segmentation of ERC and TEC was performed on an scan with a Type IIa CoS variant of regular depth $(1.30 \mathrm{~cm})$. To generate labels from the Desikan-Killiany atlas, FreeSurfer 6.0 was run on the same scan. The functional MRI atlas was provided on a subject with Type IIa CoS variant of regular depth (1.20 $\mathrm{cm}$ ) (Maass et al., 2015). The ex vivo MRI atlas was provided on a subject with a Type IIb CoS variant of shallow depth $(0.75$ $\mathrm{cm})$. For each atlas, we manually segmented the ERC plus TEC from the structural MRI following the same protocol as for our subjects. In the ex vivo case, since the CoS was shallow, the TEC was extended to the lateral bank of the CoS, as seen in histology (Insausti et al., 1998; Ding and Van Hoesen, 2010). We then mapped the atlas labels to the manually-defined ERC plus TEC surface by linear interpolation. Finally, we mapped these surfaces and their labels to the population template surface following the LDDMM framework (Beg et al., 2005). The result was four sets of labels, one from each atlas, on each vertex of the population template surface.

The Desikan-Killiany atlas defined the anterior boundary of $\mathrm{ERC}$ at the rostral end of the CoS; this was approximately $6 \mathrm{~mm}$ anterior to the boundary defined in our protocol. The functional MRI atlas defined the anterior boundary at the rostral end of the amygdala, which coincided with our protocol's boundary. The anterior boundary on the ex vivo MRI atlas was $0.5 \mathrm{~mm}$ posterior to our protocol's boundary.

The Desikan-Killiany atlas defined the posterior boundary at the caudal end of the amygdala. This excludes a posterior portion 
of the ERC that runs lateral to the hippocampal formation (Krimer et al., 1997; Insausti et al., 1998; Ding and Van Hoesen, 2010). The functional MRI atlas defined the posterior boundary as extending to the caudal end of the CoS. Since this atlas was a Type IIa CoS variant, the caudal extent of the CoS coincided with $1.2 \mathrm{~mm}$ posterior to the GI, or one $0.6 \mathrm{~mm}$ slice anterior to our protocol's boundary. The posterior boundary of the ex vivo atlas was $1.0 \mathrm{~mm}$ anterior to our protocol's boundary.

The Desikan-Killiany atlas, functional MRI atlas, and our protocol defined the medial boundary at the furthest extent where gray/white boundary was visible. The ex vivo atlas included the dorsal medial aspect of the ERC that borders the amygdala, a border that is not always visible on 3T T1 MRI. An overlay of the ex vivo atlas ERC partition and our protocol's ERC plus TEC partition highlights this difference as shown in Figure 3.

Finally, the Desikan-Killiany atlas defined the lateral extent of entorhinal cortex as the most lateral extent of the CoS. This boundary definition most closely followed that of a shallow CoS variant (Insausti et al., 1998), often seen in Type IIb CoS that were excluded from this study. The functional MRI atlas delineated the ERC ending at the shoulder of the CoS, a definition that most closely followed the boundary of a deep CoS variant (Insausti et al., 1998). Our protocol also followed the delineation that matches a deep CoS variant. Since the subjects included in this study range from regular CoS depth $(1.0-1.5 \mathrm{~cm})$ to deep CoS depth $(>1.5 \mathrm{~cm})$, it is likely that our protocol may include a small portion of the ERC within the TEC label.

\subsection{Cortical Thickness}

To calculate vertex-wise cortical thickness, we followed an established procedure based on LDDMM (Ratnanather et al., 2019). The ERC plus TEC surface was cut into two surfaces: the pial surface and the gray matter-white matter boundary surface. The gray matter-white matter boundary surface was deformed to the pial surface within the LDDMM framework, with an additional imposed constraint that the surface must flow in the direction normal to its evolving surface. Cortical thickness was then estimated as the distance along these trajectories. Average ERC thickness and average TEC thickness were calculated as the mean thickness across vertex labeled ERC and TEC, respected, as mapped from the manual structural MRI atlas.

\subsection{Group Difference Analysis}

We tested where there were differences in shape measures by diagnostic group. The log-linear mixed effects model under the null hypothesis can be written as Equation (1) given a subject $i$, scan $j$, and vertex $k$.

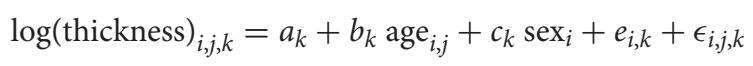

The constants $a, b, c$, and the variance of 0 -mean Gaussians $e$ and $\epsilon$ are estimated by maximum likelihood. $e$ is the subject-specific random effect, while age and sex (a binary indicator variable) are fixed effects.
The log-linear mixed effects model under the alternative hypothesis can be written as Equation (2).

$$
\begin{aligned}
\log (\text { thickness })_{i, j, k}= & a_{k}+b_{k} \text { age }_{i, j} \\
+ & \left(a_{k}^{\prime}+b_{k}^{\prime}\left(\text { age }_{i, j}-\text { age_MCIonset }_{i}\right)\right) \text { isMCI }_{i} \\
& +c \operatorname{sex}_{i}+e_{i, k}+\epsilon_{i, j, k}
\end{aligned}
$$

isMCI is a binary indicator variable for whether a subject belongs to the group that converted from NC to MCI, and age_MCIonset is the age of MCI diagnosis. $a^{\prime}$ is the mean difference in log thickness at the time of MCI diagnosis for subjects that converted from NC to MCI. $b^{\prime}$ corresponds to the disease-related rate of change in this group.

We tested whether the model under the alternative hypothesis significantly fit the data better than the model under the null hypothesis by using the likelihood ratio as a test statistic and bootstrap resampling 10,000 samples. The bootstrapped samples were constructed by sampling from whitened residuals under the null hypothesis. We compared the likelihood ratio to the distribution of likelihood ratios of the bootstrapped samples. We corrected for multiple comparisons over the vertices by using the maximum test statistic over all vertices for each set of bootstrapped samples (Nichols and Hayasaka, 2003). We rejected the null hypothesis when the true likelihood ratio was greater than $95 \%$ of the bootstrapped likelihood ratios.

\subsection{Change Point Analysis}

We tested when a change in atrophy rate occurred with respect to MCI diagnosis. Details for constructing and testing this change point model are described in another work (Tang et al., 2017). Here we provide a brief summary of the approach.

The log-linear mixed effects model under the null hypothesis can be written as Equation (3) given a subject $i$, scan $j$, and location $k$.

$$
\begin{array}{r}
\log (\text { thickness })_{i, j, k}=a_{k}+b_{k} \text { age }_{i, j}+c_{k} \text { sex }_{i} \\
+d_{k} \text { age_MCIonset }_{i}+e_{i, k}+\epsilon_{i, j, k}
\end{array}
$$

The constants $a, b, c, d$, and the variance of 0 -mean Gaussians $e$ and $\epsilon$ are estimated by maximum likelihood. $e$ is the subjectspecific random effect, while age and sex (a binary indicator variable) are fixed effects. Unlike in the group-wise difference analysis, this model has only two locations in order to reduce computational complexity: one for average ERC thickness, and the other for average TEC thickness.

The model under the alternative hypothesis can be written as Equation (4).

$$
\begin{aligned}
\log (\text { thickness })_{i, j, k}= & a_{k}+b_{k} \text { age }_{i, j} \\
& +b_{k}^{\prime}\left(\operatorname{age}_{i, j}-\left(\text { age_MCIonset }_{i}+\Delta\right)\right)^{+} \\
& +c_{k} \text { sex }_{i}+d_{k} \text { age_MCIonset }_{i}+e_{i, k}+\epsilon_{i, j, k}
\end{aligned}
$$

$\Delta$ is the number of years from a diagnosis of MCI to the change point in atrophy rate, and (age_MCIonset $\left.{ }_{i}+\Delta\right)^{+}=$ 


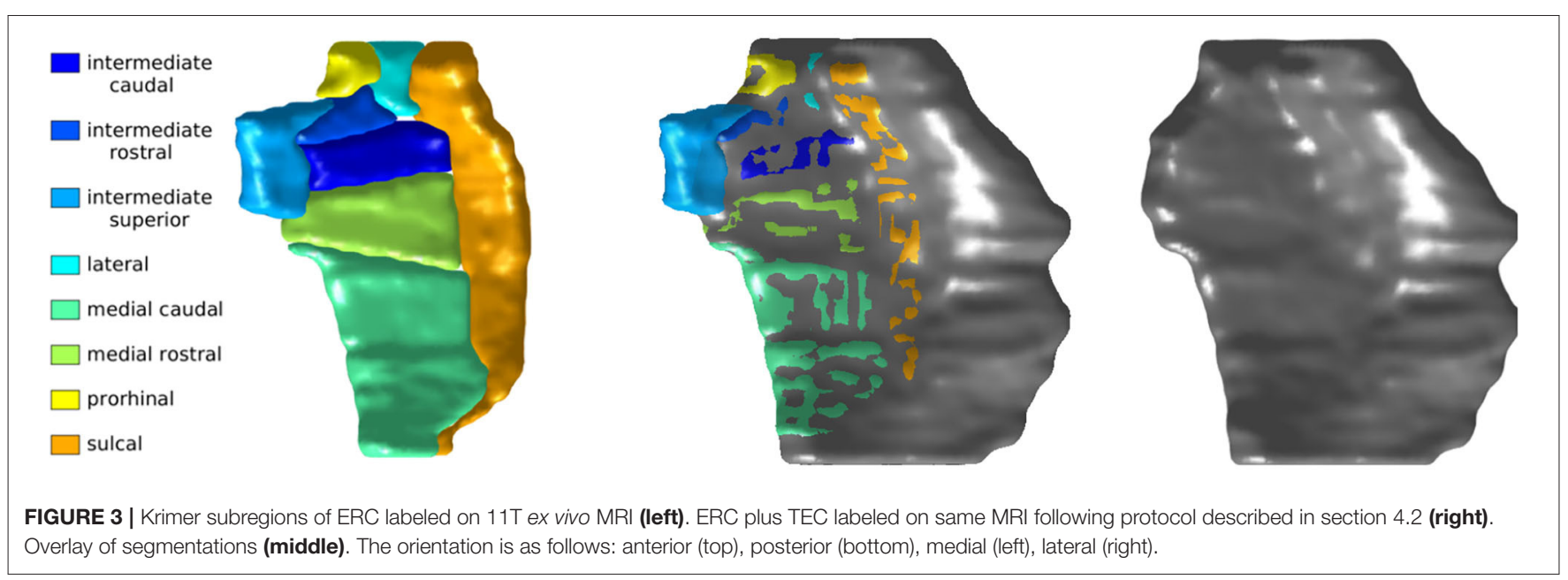

$\max \left(\right.$ age_MCIonset $\left.{ }_{i}+\Delta, 0\right)$ is the number of years past the change point. The constants $a, b, c, d$, and the variance of 0 -mean Gaussians $e$ and $\epsilon$ are estimated by maximum likelihood over a fixed $\Delta$ with yearly increments of $\Delta$ between -50 and 50 years. The best candidate $\Delta$ is calculated from the posterior mean.

For stable NC subjects, we estimated MCI diagnosis from a conditional probability distribution where the age of onset was constrained to be after the last diagnostic evaluation, and drawn from a Gaussian distribution with mean age of $\mu_{1}=93$ years and standard deviation of $\sigma_{1}=14.5$ years. This distribution of MCI diagnosis was estimated (Tang et al., 2017) using a set of 1,000 subjects enrolled with normal cognition and a family history of Alzheimer's disease.

Since the subjects in this study were selected to meet diagnostic group criteria and do not represent a random sample over the progression of the disease, we re-weighted the likelihood function by the distribution of stable $\mathrm{NC}$ and NC to MCI converters expected if the subjects were selected blind to diagnostic group. The subjects in the ADNI database were enrolled to meet a set number of subjects per diagnostic group, and as such, cannot be used to estimate this distribution. Instead, we examined the BIOCARD database, where subjects were enrolled cognitively normal and followed for up to 22 years at time we examined this database (biocard-se.org). This distribution was calculated from a subset of subjects over 65 years of age at their most recent follow-up visit. Specifically, the proportion was $184 / 260$ stable NC and $44 / 260 \mathrm{NC}$ to MCI converters.

We tested whether the model under the alternative hypothesis significantly fit the data better than the model under the null hypothesis using the likelihood ratio as a test statistic and bootstrap resampling 1,000 samples. The bootstrapped samples are constructed by sampling from whitened residuals under the null hypothesis, with imputed values for age_MCIonset for stable NC subjects. We compared the likelihood ratio to the distribution of likelihood ratios of the bootstrapped samples and rejected the null hypothesis when the likelihood ratio was greater than $95 \%$ of the bootstrapped likelihood ratios. We used the maximum test statistic over pairs of ERC and TEC statistics to control family-wise error rate (Nichols and Hayasaka, 2003).

In the case where the null hypothesis was rejected, we then determined the confidence interval for $\Delta, b$ and $b^{\prime}$ by bootstrap resampling under the alternative hypothesis 1,000 times. The bootstrapped samples were constructed by sampling from whitened residuals under the alternative hypothesis, with imputed values for age_MCIonset for NC subjects. In the case where the null hypothesis was rejected for both ERC and TEC measures, we then calculated the probability that the change point for TEC occurred before the change point for ERC based on the change point $\Delta$ of each pair of bootstrapped samples under the alternative hypothesis. This one-sided test was selected based on histological evidence that changes occurred in the TEC before the ERC (Braak et al., 2006).

\section{RESULTS}

\subsection{Surface Parcellation}

The results of the four atlas mappings onto the population template surface are shown in Figure 4. Comparison of the atlases highlight inconsistencies introduced by varying nomenclature and $\mathrm{CoS}$ variant. The automated labels based on cortical folding used by Desikan-Killany defined the ERC extending into the $\mathrm{CoS}$, a definition that matches a shallow $\mathrm{CoS}$ variant. The atlas also excluded the posterior extent of the ERC. In contrast, the functional MRI atlas defined the perirhinal cortex (PRC), an area that includes the TEC, and separated this structure from the ERC using a definition that matches a deep CoS variant. Histologically-defined subregions of the ERC show yet another popular parcellation of this region on a regular depth $(1-1.5 \mathrm{~cm})$ $\mathrm{CoS}$ variant. Note how in this definition, the sulcal ERC extends into the shoulder of the CoS. In this study, average ERC and average TEC metrics were calculated based on the labels shown in the manual structural labels (top left).

\subsection{Cortical Thickness}

Average cortical thickness of TEC and average cortical thickness of ERC are plotted in Figure 5. The TEC is slightly thicker than 


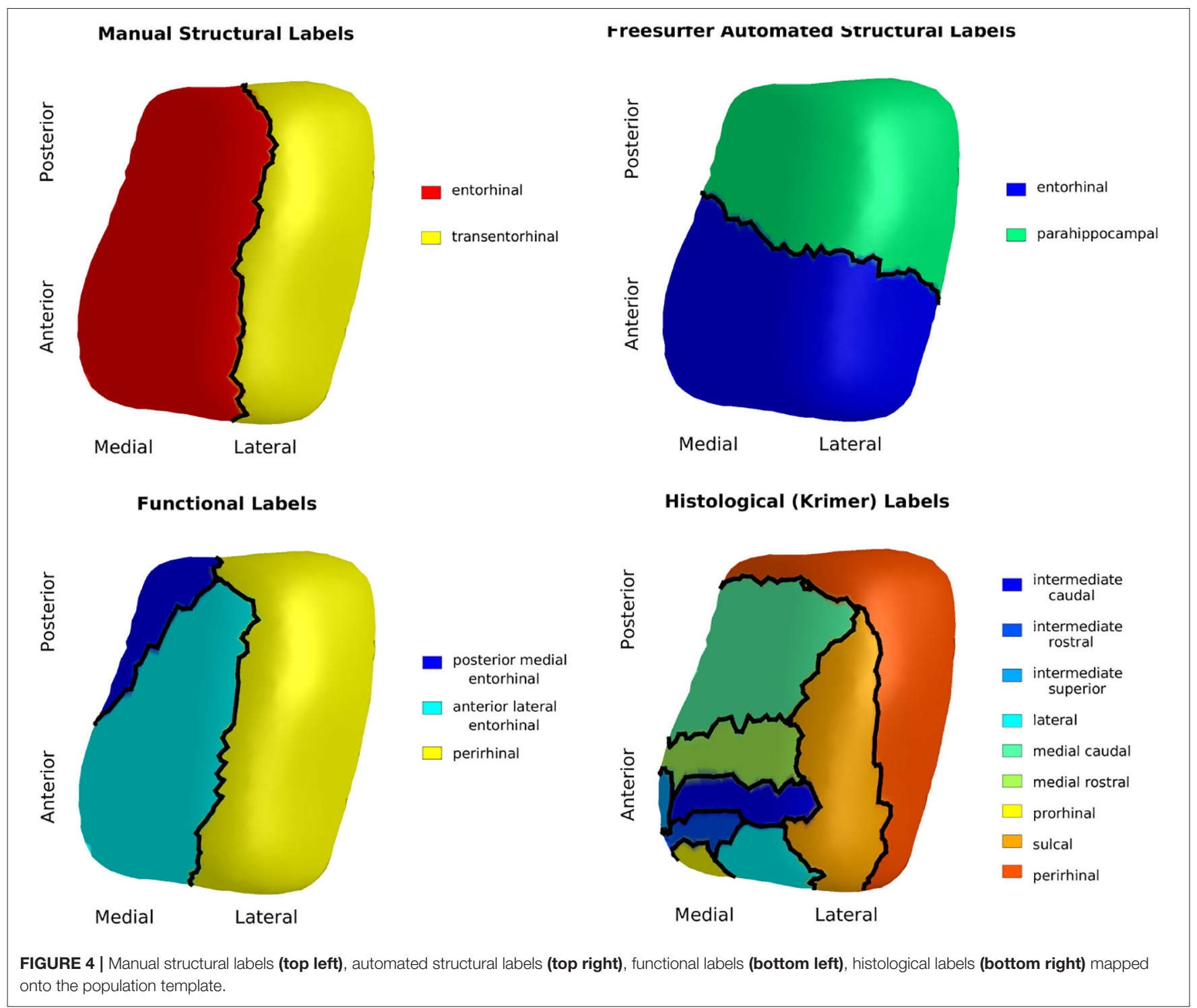

the ERC, which is in agreement with previous work (Kulason et al., 2019). In both regions, organization by MCI diagnosis date show cortical thickness measures decrease with progression of the disease, and that the rate of cortical thinning is noticeably steeper in NC to MCI converters than in stable NC subjects.

\subsection{Diagnostic Group Difference Analysis}

We rejected the null hypothesis with global $p<0.0001$ and concluded that there was a difference in cortical thickness between diagnostic groups. Figure 6 shows the difference in atrophy and atrophy rate across all vertices. A summary of average and maximum atrophy/atrophy rates is shown in Table 3. As the data demonstrates, at the time of MCI diagnosis, individuals who had progressed to a diagnosis of MCI were as much as $0.58 \mathrm{~mm}$ thinner in the ERC and $0.60 \mathrm{~mm}$ thinner in the TEC. The additional atrophy rate in the participants who progressed from NC to MCI was $2.96 \%$ per year in the ERC on average and $2.43 \%$ per year in the TEC on average. This is a notable increase from age-related atrophy which was, on average, $0.68 \%$ per year in the TEC and $0.66 \%$ per year in the ERC. In other words, the average total atrophy rate in $\mathrm{NC}$ to MCI converters was 5.35 times greater in the ERC and 4.68 times greater in the TEC compared to stable NC subjects.

\subsection{Change Point Analysis}

We rejected the null hypothesis and concluded that there was a change point 9.02 years before MCI onset for the ERC $(p<$ $0.001)$ and 10.69 years before MCI diagnosis for the TEC $(p<$ 0.001 ). Prior to the change point, the atrophy rate was $0.35 \% /$ year for the ERC and $0.34 \% / y e a r$ for the TEC. After the change point, the additional atrophy was $3.75 \%$ /year for the ERC and $2.58 \% / y e a r$ for the TEC. The $95 \%$ confidence interval for the parameters of interest are shown in Table 4. The ERC change point in thickness occurred at or before the TEC change point 

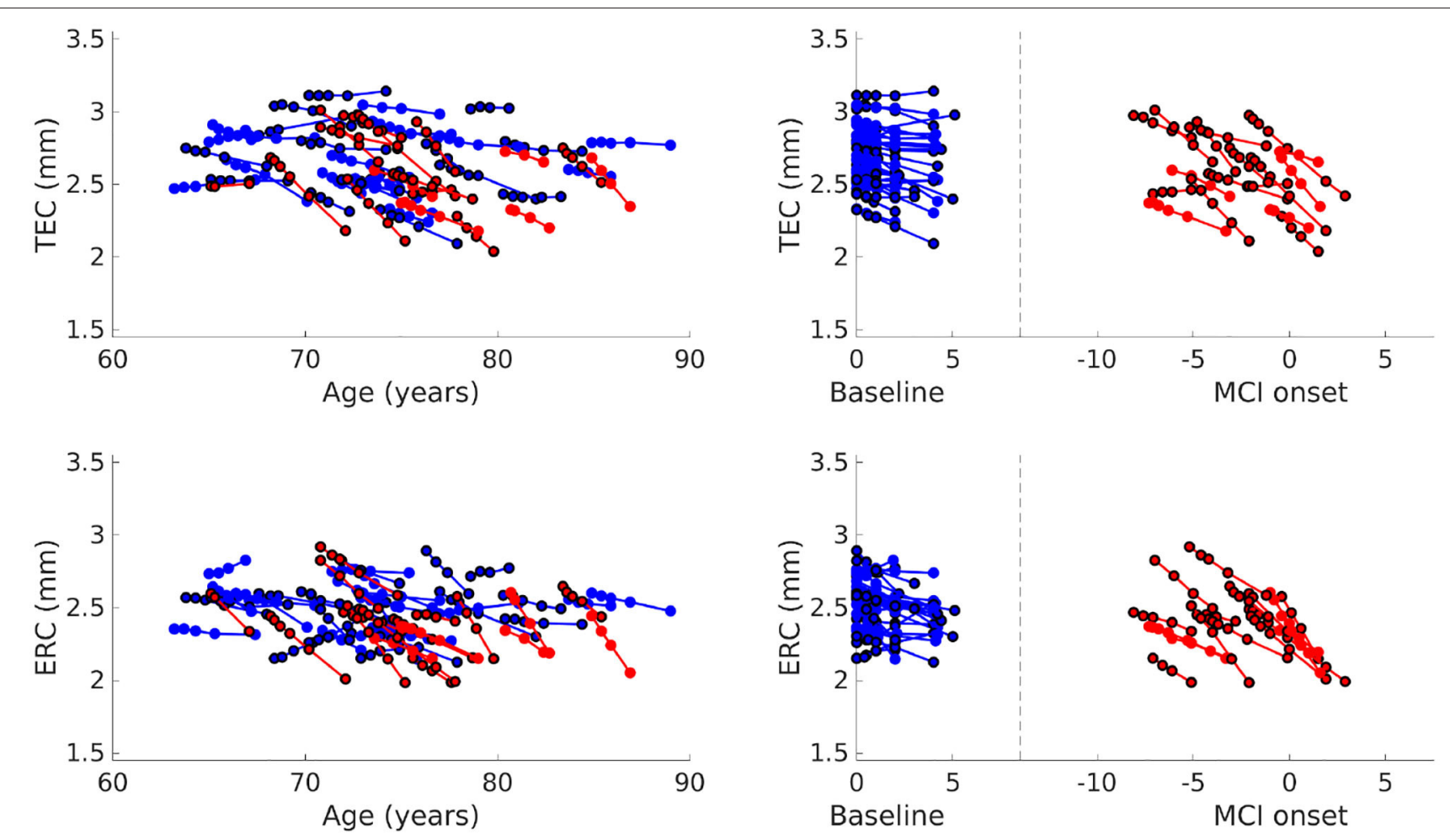

FIGURE 5 | Average TEC thickness (top) and ERC thickness (bottom) plotted over age (left). Average TEC thickness (top) and ERC thickness (bottom) plotted with respect to time from baseline scan for $\mathrm{NC}$, and with respect to time from $\mathrm{MCl}$ onset for $\mathrm{NC}$ to $\mathrm{MCl}$ converters (right). Each line corresponds to a subject, the color to a diagnostic group (stable NC, NC to MCl), and the marker border to sex (no border is male, bordered is female).

TABLE 3 | Summary of group-wise difference analysis by region.

\begin{tabular}{lcccc}
\hline & $\begin{array}{c}\text { ERC } \\
\text { average }\end{array}$ & $\begin{array}{c}\text { ERC } \\
\max \end{array}$ & $\begin{array}{c}\text { TEC } \\
\text { average }\end{array}$ & $\begin{array}{c}\text { TEC } \\
\max \end{array}$ \\
\hline Atrophy at & & & & \\
MCl diagnosis & $0.25 \mathrm{~mm}$ & $\begin{array}{l}0.58 \mathrm{~mm} \\
(8.46 \%)\end{array}$ & $\begin{array}{c}0.23 \mathrm{~mm} \\
(16.54 \%)\end{array}$ & $\begin{array}{l}(7.63 \%) \\
(17.34 \%)\end{array}$ \\
$\begin{array}{l}\text { MCl-related } \\
\text { Atrophy rate }\end{array}$ & $2.96 \% /$ year & $4.23 \% /$ year & $2.43 \% /$ year & $4.11 \% /$ year \\
age-related & & & & \\
Atrophy rate & $0.68 \% /$ year & $2.08 \% /$ year & $0.66 \% /$ year & $1.61 \% /$ year
\end{tabular}

in $3.75 \%$ of bootstrapped samples. We concluded that the TEC change point preceded the ERC change point.

\section{DISCUSSION}

The first major finding of this study is that anterior regions of the ERC and TEC were more than half a millimeter thinner (up to $17 \%$ thinner) in NC to MCI converters at the time of MCI diagnosis, and that disease-related atrophy was roughly $3 \%$ per year. The evidence suggests that disease-related atrophy begins prior to an MCI diagnosis in the anterior lateral region of ERC and anterior region of TEC. This is in line with our previous study
TABLE 4 | 95\% confidence interval (min, max).

\begin{tabular}{lccc}
\hline & $\begin{array}{c}\text { Change point } \\
\text { (years before } \mathbf{M C l})\end{array}$ & $\begin{array}{c}\text { Age-related rate } \\
\text { (\%/year atrophy) }\end{array}$ & $\begin{array}{c}\text { Disease-related rate } \\
\text { (\%/year atrophy) }\end{array}$ \\
\hline ERC thickness & $(7.63,11.31)$ & $(0.07,0.65)$ & $(3.03,4.41)$ \\
TEC thickness & $(8.92,13.80)$ & $(0.10,0.56)$ & $(2.11,3.08)$ \\
\hline
\end{tabular}

Change point, age-related rate, and disease-related rate correspond to the variables $-\Delta$, $-b$, and $-b^{\prime}$, respectively. The disease-related rate is the additional rate seen post change point.

that examined subjects after an MCI diagnosis, where we found disease-related thickness atrophy was 5\% atrophy per year in the TEC and that MCI subjects were on average 23\% thinner than NC (Kulason et al., 2019). The 3\% atrophy per year in NC to MCI converters vs. $5 \%$ atrophy per year in subjects after MCI diagnosis may suggest that the atrophy rate increases with progression of the disease from the preclinical to clinical stage.

The second major finding of this study is that there was a change in the rate of ERC thickness atrophy 8-11 years prior to MCI diagnosis, and a change in the rate of TEC thickness atrophy 9-14 years before the diagnosis of MCI. The order of the change points, TEC before ERC, is consistent with histological report of neurofibrillary tau accumulation in this region (Braak et al., 2006). The time of change point is consistent with a 


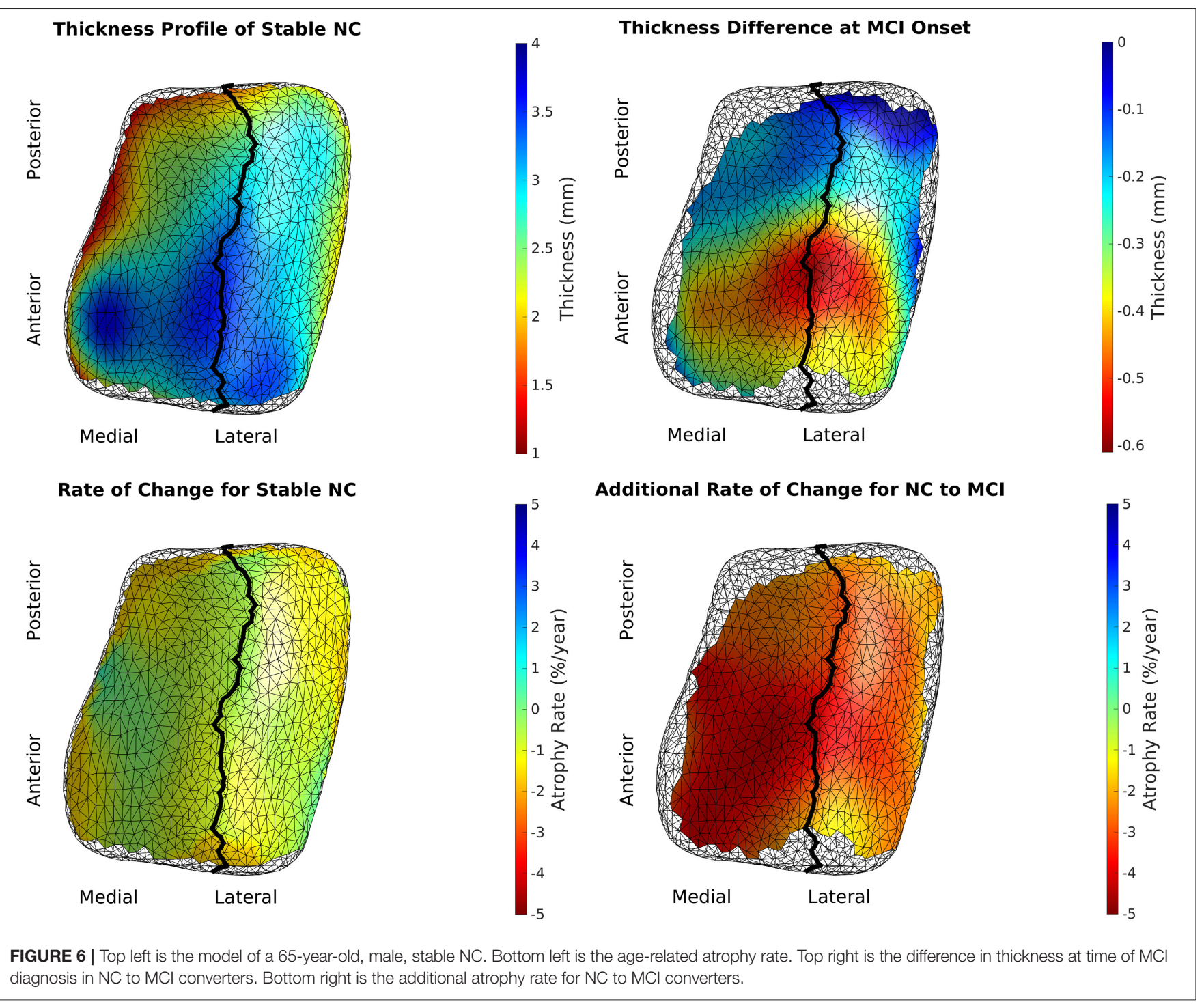

previous study, which found changes in surface area of the FreeSurfer-defined ERC 8-10 years prior to symptom onset (Younes et al., 2014).

These findings emphasize the discrepancies in nomenclature pertaining to the TEC and ERC. We showed how the DesikanKilliany atlas defined ERC beginning anterior to our protocol and ending anterior to our protocol. Our protocol closely matched the anterior and posterior boundaries defined in the function MRI atlas and ex vivo atlases. The Desikan-Killiany ERC extended laterally into the CoS, which is accurate for shallow CoS common variant in Type IIb CoS, and not as accurate for the regular and deep CoS variants that were included in this study. The ex vivo atlas, a subject with Type IIa CoS variant of regular depth, marked the sulcal ERC lateral boundary shortly past the shoulder of the $\mathrm{CoS}$, which is consistent with cytoarchitectonically-defined ERC in previous studies (Krimer et al., 1997; Insausti et al., 1998; Ding and Van Hoesen, 2010). The functional MRI atlas and Desikan-Killiany atlas chose not to define the TEC separate from perirhinal cortex and fusiform gyrus, respectively. The 4 surface parcellations mapped to the same coordinate system highlight the need for a standardized nomenclature of this region, much like the work being done to standardize sub-regional boundaries of the hippocampus (Adler et al., 2018; Olsen et al., 2019).

There are a number of strengths to this study. The subjects were carefully selected to (1) follow a strict set of inclusion criteria for diagnostic grouping, and (2) exclude subjects with a discontinuous $\mathrm{CoS}$ within the region of interest. This was done to reduce confounding factors introduced by other medical conditions and natural variability in cortical folding. In addition, scans were manually segmented to avoid errors introduced by automated segmentation methods. Finally, the results were put in the context of several atlases for broader interpretation of findings.

There are also a few limitations to this study, the first being a relatively small sample size. Accurate segmentations, which have been performed manually for this study, are extremely 
time consuming. The ERC is particularly difficult to segment automatically due to its proximity with the meninges and oculomotor nerve. These neighboring structures are a similar intensity to gray matter voxels in $\mathrm{T} 1$ scans. Some recent work has been done to address this issue using automatic parcellation (Xie et al., 2017); a future direction is to determine whether this type of automated approach to segmentation affects ERC and TEC metrics produced in this analysis.

Another limitation of this study is that the distribution of samples is biased by diagnostic grouping. It is difficult to estimate the true distribution of diagnostic groups because the ADNI protocol selected subjects based on diagnosis and the follow-up time varies. This is mitigated by the use of distribution estimates calculated from the BIOCARD database.

In the future, this study can be extended to include shallow, discontinuous $\operatorname{CoS}$ variants for detecting Alzheimer's-related changes. It is of interest to develop metrics of disease progression that are robust to this natural variation in folding. Autopsy studies have shown that subjects with a shallow, discontinuous $\mathrm{CoS}$ have a TEC that begins at the deepest extent of the CoS and extends out laterally, whereas deep, continuous CoS have a TEC that begins at the shoulder of the CoS and extends only to the deepest extent of the CoS (Insausti et al., 1998; Ding and Van Hoesen, 2010). Therefore, a multi-atlas approach with CoS variant-specific atlases may be desirable to delineate the ERC and TEC accurately for a full population of subjects.

Finally, given the localization of tau to CA1 after initial deposits along the boundary of TEC and ERC (Braak and Braak, 1991), it is of interest to extend thickness analysis to this region. Unfortunately, the CA1 subfield cannot be segmented separately from the hippocampal formation in 3T T1 MRI. Previous volumetric analysis of the hippocampus has shown $9 \%$ atrophy in MCI subjects, compared to $27 \%$ volumetric atrophy of ERC plus TEC in MCI subjects (Kulason et al., 2019). Previous change point analysis of surface expansion/contraction metrics have shown a hippocampal change point 2-4 years prior to symptom onset, compared to 8-10 years prior to symptom onset for a Desikan-Killiany defined ERC (Younes et al., 2014). As more high resolution T2 MRI data become available, such as with data being collected in the more recent ADNI 3 protocol, it will be of interest to extend this thickness analysis to the CA1 subfield.

This study provides strong evidence that TEC and ERC thickness is a sensitive measure to progression to the symptomatic phase of Alzheimer's disease and that disease-related atrophy begins to occur at least 9 years prior to a clinical diagnosis of MCI.

\section{DATA AVAILABILITY STATEMENT}

The data analyzed in this study is subject to the following licenses/restrictions: Individuals need to register with ADNI and agree to the conditions in the "ADNI Data Use Agreement" and undergo limited screening by the DPC before accessing data. Requests to access these datasets should be directed to http://adni. loni.usc.edu/data-samples/access-data/.

\section{ETHICS STATEMENT}

The studies involving human participants were reviewed and approved by Good Clinical Practice guidelines, the Declaration of Helsinki, and the US 21 CFR Part 50-Protection of Human Subjects, and Part 56-Institutional Review Boards (IRB). The patients/participants provided their written informed consent to participate in this study.

\section{AUTHOR CONTRIBUTIONS}

SK and EX processed the data. SK performed the analysis, drafted the manuscript, and designed the figures. DT, MA, LY, and MM contributed to the design and implementation of the research and to the analysis of the results. All authors contributed to the editing of the manuscript.

\section{FUNDING}

This work was supported by the National Institutes of Health (P41-EB015909 and R01-EB020062); and the Kavli Neuroscience Discovery Institute. MM reports personal fees from AnatomyWorks, LLC, outside the submitted work, and jointly owns AnatomyWorks. Dr. Miller's relationship with AnatomyWorks is being handled under full disclosure by the Johns Hopkins University.

\section{ACKNOWLEDGMENTS}

A special thank you to Kanami Mori for her work on manually segmenting the $11 \mathrm{~T}$ ex vivo MRI atlas and to Anthony Kolasny for maintaining the Center for Imaging Science server on which much of this analysis was run.

Data used in the preparation of this article were obtained from the Alzheimer's Disease Neuroimaging Initiative (ADNI) database (adni.loni.usc.edu). As such, the investigators within the ADNI contributed to the design and implementation of ADNI and/or provided data but did not participate in the analysis or writing of this report. A complete listing of ADNI investigators can be found at http://adni.loni.usc.edu/wpcontent/uploads/how_to_apply/ADNI_Acknowledgement_List. pdf. Data collection and sharing for this project was funded by the Alzheimer's Disease Neuroimaging Initiative (ADNI) (National Institutes of Health Grant U01 AG024904) and DODADNI (Department of Defense award number W81XWH-12-2-0012). ADNI is funded by the National Institute on Aging, the National Institute of Biomedical Imaging and Bioengineering, and through generous contributions from the following: AbbVie, Alzheimer's Association; Alzheimer's Drug Discovery Foundation; Araclon Biotech; BioClinica, Inc.; Biogen; BristolMyers Squibb Company; CereSpir, Inc.; Cogstate; Eisai Inc.; Elan Pharmaceuticals, Inc.; Eli Lilly and Company; EuroImmun; F. Hoffmann-La Roche Ltd. and its affiliated company Genentech, Inc.; Fujirebio; GE Healthcare; IXICO Ltd.; Janssen Alzheimer Immunotherapy Research \& Development, LLC; Johnson \& Johnson Pharmaceutical Research \& Development LLC; Lumosity; Lundbeck; Merck \& Co, Inc.; Meso Scale Diagnostics, 
LLC; NeuroRx Research; Neurotrack Technologies; Novartis Pharmaceuticals Corporation; Pfizer Inc.; Piramal Imaging; Servier; Takeda Pharmaceutical Company; and Transition Therapeutics. The Canadian Institutes of Health Research is providing funds to support ADNI clinical sites in Canada. Private sector contributions are facilitated by the Foundation for the National Institutes of Health (www.fnih.org). The grantee organization is the Northern California Institute for Research and Education, and the study is coordinated by the Alzheimer's Therapeutic Research Institute at the University of Southern California. ADNI data are disseminated by the Laboratory for Neuroimaging at the University of Southern California.

Data from the BIOCARD study are supported by grant U19 AG033655 from the National Institute on Aging. The BIOCARD Study consists of 7 Cores with the following members: (1) The Administrative Core (MA, Barbara Rodzon), (2) the Clinical Core (MA, Anja Soldan, Rebecca Gottesman, Ned Sacktor, Corinne Pettigrew, Scott Turner, Leonie Farrington, Maura Grega, Jules, Gilles, Gay Rudow, Rostislav Brichko, Scott Rudow), (3) the Imaging Core (MM, Susumu Mori, Tilak Ratnanather, Anthony Kolasny, Hanzhang Lu, Kenichi Oishi, LY), (4) the Biospecimen Core (Abhay Moghekar, Jacqueline Darrow, Richard O’Brien), (5) the Informatics Core (Roberta

\section{REFERENCES}

Adler, D. H., Wisse, L. E., Ittyerah, R., Pluta, J. B., Ding, S.-L., Xie, L., et al. (2018). Characterizing the human hippocampus in aging and Alzheimer's disease using a computational atlas derived from ex vivo MRI and histology. Proc. Natl. Acad. Sci. U.S.A. 115, 4252-4257. doi: 10.1073/pnas.1801093115

Apostolova, L. G., Mosconi, L., Thompson, P. M., Green, A. E., Hwang, K. S., Ramirez, A., et al. (2010). Subregional hippocampal atrophy predicts Alzheimer's dementia in the cognitively normal. Neurobiol. Aging 31, 1077-1088. doi: 10.1016/j.neurobiolaging.2008.08.008

Atiya, M., Hyman, B. T., Albert, M. S., and Killiany, R. (2003). Structural magnetic resonance imaging in established and prodromal Alzheimer disease: a review. Alzheimer Dis. Assoc. Disord. 17, 177-195. doi: 10.1097/00002093-200307000-00010

Beg, M. F., Miller, M. I., Trouvé, A., and Younes, L. (2005). Computing large deformation metric mappings via geodesic flows of diffeomorphisms. Int. J. Comput. Vis. 61, 139-157. doi: 10.1023/B:VISI.0000043755.93987.aa

Braak, H., Alafuzoff, I., Arzberger, T., Kretzschmar, H., and Del Tredici, K. (2006). Staging of Alzheimer disease-associated neurofibrillary pathology using paraffin sections and immunocytochemistry. Acta Neuropathol. 112, 389-404. doi: 10.1007/s00401-006-0127-z

Braak, H., and Braak, E. (1991). Neuropathological stageing of Alzheimer-related changes. Acta Neuropathol. 4, 239-259. doi: 10.1007/BF00308809

Center for Integrative Biomedical Computing (2016). Seg3D: Volumetric Image Segmentation and Visualization. Scientific Computing and Imaging Institute (SCI). Available online at: http://www.seg3d.org

Csernansky, J. G., Wang, L., Swank, J., Miller, J. P., Gado, M., McKeel, D., et al. (2005). Preclinical detection of Alzheimer's disease: hippocampal shape and volume predict dementia onset in the elderly. Neuroimage 25, 783-792. doi: 10.1016/j.neuroimage.2004.12.036

den Heijer, T., Geerlings, M. I., Hoebeek, F. E., Hofman, A., Koudstaal, P. J., and Breteler, M. M. (2006). Use of hippocampal and amygdalar volumes on magnetic resonance imaging to predict dementia in cognitively intact elderly people. Arch. Gen. Psychiatry 63, 57-62. doi: 10.1001/archpsyc.63.1.57

Desikan, R. S., Ségonne, F., Fischl, B., Quinn, B. T., Dickerson, B. C., Blacker, D., et al. (2006). An automated labeling system for subdividing the human
Scherer, David Shade, Ann Ervin, Jennifer Jones, Hamadou Coulibaly, Kathy Moser), the (6) Biostatistics Core (Mei-Cheng Wang, Yuxin Zhu, Jiangxia Wang), and (7) the Neuropathology Core (Juan Troncoso, Olga Pletnikova, Karen Fisher). We would also like to acknowledge the members of the BIOCARD Scientific Advisory Board who provide oversight an guidance regarding the conduct of the study including: Drs. John Csernansky, David Holtzman, David Knopman, Walter Kukull and Kevin Duff, as well as Drs. Laurie Ryan and John Hsiao, who provide oversight on behalf of the NIA. Additionally, we recognize the members of the BIOCARD Resource Allocation Committee who provide ongoing guidance regarding the use of the biospecimens collected as part of the study, including: Drs. Constantine Lyketsos, Carlos Pardo, Gerard Schellenberg, Leslie Shaw, Madhav Thambisetty, and John Trojanowski. We would like to acknowledge the contributions of the Geriatric Psychiatry Branch (GPB) of the intramural program of the NIMH who initiated this study (PI: Dr. Trey Sunderland). Dr. Karen Putnam provided documentation of the GPB study procedures and the datafiles received from NIMH. We acknowledge the altruism of the participants and their families and contributions of the BIOCARD research and support staff for their contributions to this study. cerebral cortex on MRI scans into gyral based regions of interest. Neuroimage 31, 968-980. doi: 10.1016/j.neuroimage.2006.01.021

Devanand, D. P., Pradhaban, G., Liu, X., Khandji, A., De Santi, S., Segal, S., et al. (2007). Hippocampal and entorhinal atrophy in mild cognitive impairment prediction of Alzheimer disease. Neurology 68, 828-836. doi: 10.1212/01.wnl.0000256697.20968.d7

Dickerson, B., Stoub, T., Shah, R., Sperling, R., Killiany, R., Albert, M., et al. (2011). Alzheimer-signature MRI biomarker predicts AD dementia in cognitively normal adults. Neurology 76, 1395-1402. doi: 10.1212/WNL.0b013e3182166e96

Ding, S.-L., and Van Hoesen, G. W. (2010). Borders, extent, and topography of human perirhinal cortex as revealed using multiple modern neuroanatomical and pathological markers. Hum. Brain Mapp. 31, 1359-1379. doi: 10.1002/hbm.20940

Durrleman, S., Fletcher, T., Gerig, G., and Niethammer, M. (2012). "Spatio-temporal image analysis for longitudinal and time-series image data," in Second International Workshop, STIA 2012, Held in Conjunction with MICCAI 2012 (Nice: Springer). doi: 10.1007/978-3-64233555-6

Huntgeburth, S. C., and Petrides, M. (2012). Morphological patterns of the collateral sulcus in the human brain. Eur. J. Neurosci. 35, 1295-1311. doi: 10.1111/j.1460-9568.2012.08031.x

Insausti, R., Juottonen, K., Soininen, H., Insausti, A. M., Partanen, K., Vainio, P., et al. (1998). MR volumetric analysis of the human entorhinal, perirhinal, and temporopolar cortices. Am. J. Neuroradiol. 19, 659-671. doi: 10.1016/S0197-4580(98)00007-4

Jack, C. R., Shiung, M. M., Gunter, J. L., O’brien, P. C., Weigand, S. D., Knopman, D. S., et al. (2004). Comparison of different MRI brain atrophy rate measures with clinical disease progression in AD. Neurology 62, 591-600. doi: 10.1212/01.WNL.0000110315.26026.EF

Kantarci, K., and Jack, C. R. (2003). Neuroimaging in Alzheimer disease: an evidence-based review. Neuroimaging Clin. 13, 197-209. doi: 10.1016/S1052-5149(03)00025-X

Kantarci, K., and Jack, C. R. (2004). Quantitative magnetic resonance techniques as surrogate markers of Alzheimer's disease. NeuroRx 1, 196-205. doi: 10.1602/neurorx.1.2.196 
Krimer, L. S., Hyde, T. M., Herman, M. M., and Saunders, R. C. (1997). The entorhinal cortex: an examination of cyto-and myeloarchitectonic organization in humans. Cereb. Cortex 7, 722-731. doi: 10.1093/cercor/7.8.722

Kulason, S., Tward, D. J., Brown, T., Sicat, C. S., Liu, C.-F., Ratnanather, J. T., et al. (2019). Cortical thickness atrophy in the transentorhinal cortex in mild cognitive impairment. Neuroimage Clin. 21:101617. doi: 10.1016/j.nicl.2018.101617

La Joie, R., Perrotin, A., Barré, L., Hommet, C., Mézenge, F., Ibazizene, M., et al. (2012). Region-specific hierarchy between atrophy, hypometabolism, and $\beta$-amyloid $(\mathrm{A} \beta$ ) load in Alzheimer's disease dementia. J. Neurosci. 32, 16265-16273. doi: 10.1523/JNEUROSCI.217012.2012

Ma, J., Miller, M. I., Trouvé, A., and Younes, L. (2008). Bayesian template estimation in computational anatomy. Neuroimage 42, 252-261. doi: 10.1016/j.neuroimage.2008.03.056

Maass, A., Berron, D., Libby, L. A., Ranganath, C., and Düzel, E. (2015). Functional subregions of the human entorhinal cortex. elife 4:e06426. doi: $10.7554 /$ eLife.06426

Miller, M. I., Ratnanather, J. T., Tward, D. J., Brown, T., Lee, D. S., Ketcha, M., et al. (2015a). Network neurodegeneration in Alzheimer's disease via MRI based shape diffeomorphometry and high-field atlasing. Front. Bioeng. Biotechnol. 3:54. doi: 10.3389/fbioe.2015.00054

Miller, M. I., Younes, L., Ratnanather, J. T., Brown, T., Trinh, H., Lee, D. S., et al. (2015b). Amygdalar atrophy in symptomatic Alzheimer's disease based on diffeomorphometry: the BIOCARD cohort. Neurobiol. Aging 36:S3-S10. doi: 10.1016/j.neurobiolaging.2014.06.032

Miller, M. I., Younes, L., Ratnanather, J. T., Brown, T., Trinh, H., Postell, E., et al. (2013). The diffeomorphometry of temporal lobe structures in preclinical Alzheimer's disease. Neuroimage Clin. 3, 352-360. doi: 10.1016/j.nicl.2013.09.001

Nelson, P. T., Alafuzoff, I., Bigio, E. H., Bouras, C., Braak, H., Cairns, N. J., et al. (2012). Correlation of Alzheimer disease neuropathologic changes with cognitive status: a review of the literature. J. Neuropathol. Exp. Neurol. 71, 362-381. doi: 10.1097/NEN.0b013e31825018f7

Nichols, T., and Hayasaka, S. (2003). Controlling the familywise error rate in functional neuroimaging: a comparative review. Stat. Methods Med. Res. 12, 419-446. doi: 10.1191/0962280203sm341ra

Olsen, R. K., Carr, V. A., Daugherty, A. M., La Joie, R., Amaral, R. S., Amunts, K., et al. (2019). Progress update from the hippocampal subfields group. Alzheimers Dement. Diagn. Assess. Dis. Monitor. 11, 439-449. doi: 10.1016/j.dadm.2019.04.001

Pettigrew, C., Soldan, A., Zhu, Y., Wang, M.-C., Moghekar, A., Brown, T., et al. (2016). Cortical thickness in relation to clinical symptom onset in preclinical AD. Neuroimage Clin. 12, 116-122. doi: 10.1016/j.nicl.2016.06.010

Ratnanather, J. T., Arguillére, S., Kutten, K. S., Hubka, P., Kral, A., and Younes, L. (2019). "Chapter 7: 3D normal coordinate systems for cortical areas," in Mathematics of Shapes and Applications, Vol. 37 (Singapore: World Scientific), 167-180. doi: 10.1142/9789811200137_0007
Soldan, A., Pettigrew, C., Lu, Y., Wang, M.-C., Selnes, O., Albert, M., et al. (2015). Relationship of medial temporal lobe atrophy, APOE genotype, and cognitive reserve in preclinical Alzheimer's disease. Hum. Brain Mapp. 36, 2826-2841. doi: 10.1002/hbm.22810

Sperling, R. A., Aisen, P. S., Beckett, L. A., Bennett, D. A., Craft, S., Fagan, A. M., et al. (2011). Toward defining the preclinical stages of Alzheimer's disease: recommendations from the National Institute on Aging-Alzheimer's Association workgroups on diagnostic guidelines for Alzheimer's disease. Alzheimers Dement. 7, 280-292. doi: 10.1016/j.jalz.2011.03.003

Tang, X., Miller, M. I., and Younes, L. (2017). Biomarker change-point estimation with right censoring in longitudinal studies. Ann. Appl. Stat. 11:1738. doi: 10.1214/17-AOAS1056

Tward, D., Miller, M., Alzheimer's Disease Neuroimaging Initiative (2017a). "Unbiased diffeomorphic mapping of longitudinal data with simultaneous subject specific template estimation," in Graphs in Biomedical Image Analysis, Computational Anatomy and Imaging Genetics (Cham: Springer), 125-136. doi: 10.1007/978-3-319-67675-3_12

Tward, D. J., Sicat, C. S., Brown, T., Bakker, A., Gallagher, M., Albert, M., et al. (2017b). Entorhinal and transentorhinal atrophy in mild cognitive impairment using longitudinal diffeomorphometry. Alzheimers Dement. 9, 41-50. doi: 10.1016/j.dadm.2017.07.005

Wolk, D. A., Das, S. R., Mueller, S. G., Weiner, M. W., Yushkevich, P. A., Initiative, A. D. N., et al. (2017). Medial temporal lobe subregional morphometry using high resolution MRI in Alzheimer's disease. Neurobiol. Aging 49, 204-213. doi: 10.1016/j.neurobiolaging.2016.09.011

Xie, L., Das, S. R., Wisse, L. E., Ittyerah, R., Yushkevich, P. A., Wolk, D. A., et al. (2018). Early tau burden correlates with higher rate of atrophy in transentorhinal cortex. J. Alzheimers Dis. 62, 85-92. doi: 10.3233/JAD-170945

Xie, L., Pluta, J. B., Das, S. R., Wisse, L. E., Wang, H., Mancuso, L., et al. (2017). Multi-template analysis of human perirhinal cortex in brain MRI: explicitly accounting for anatomical variability. Neuroimage 144, 183-202. doi: 10.1016/j.neuroimage.2016.09.070

Younes, L., Albert, M., Miller, M. I., BIOCARD Research Team (2014). Inferring changepoint times of medial temporal lobe morphometric change in preclinical Alzheimer's disease. Neuroimage Clin. 5, 178-187. doi: 10.1016/j.nicl.2014.04.009

Conflict of Interest: The authors declare that the research was conducted in the absence of any commercial or financial relationships that could be construed as a potential conflict of interest.

Copyright (c) 2020 Kulason, Xu, Tward, Bakker, Albert, Younes, and Miller. This is an open-access article distributed under the terms of the Creative Commons Attribution License (CC BY). The use, distribution or reproduction in other forums is permitted, provided the original author(s) and the copyright owner(s) are credited and that the original publication in this journal is cited, in accordance with accepted academic practice. No use, distribution or reproduction is permitted which does not comply with these terms. 